\title{
Identification of a PKP2 gene deletion in a family with arrhythmogenic right ventricular cardiomyopathy
}

\author{
Ilena Egle Astrid Li Mura ${ }^{1}$, Barbara Bauce ${ }^{2}$, Andrea Nava ${ }^{2}$, Manuela Fanciulli $^{3}$, Giovanni Vazza ${ }^{1}$, \\ Elisa Mazzotti ${ }^{2}$, Ilaria Rigato ${ }^{2}$, Marzia De Bortoli ${ }^{1}$, Giorgia Beffagna ${ }^{1}$, Alessandra Lorenzon ${ }^{1}$, Martina Calore ${ }^{1}$, \\ Emanuela Dazzo ${ }^{1}$, Carlo Nobile ${ }^{4}$, Maria Luisa Mostacciuolo ${ }^{1}$, Domenico Corrado ${ }^{2}$, Cristina Basso ${ }^{2}$, \\ Luciano Daliento ${ }^{2}$, Gaetano Thiene $^{2}$ and Alessandra Rampazzo ${ }^{\star 1}$
}

\begin{abstract}
Arrhythmogenic right ventricular cardiomyopathy (ARVC) is a primary heart muscle disease characterized by progressive myocardial loss, with fibro-fatty replacement, and high frequency of ventricular arrhythmias that can lead to sudden cardiac death. ARVC is a genetically determined disorder, usually caused by point mutations in components of the cardiac desmosome. Conventional mutation screening of ARVC genes fails to detect causative mutations in about $50 \%$ of index cases, suggesting a further genetic heterogeneity. We performed a genome-wide linkage study and a copy number variations (CNVs) analysis, using high - density SNP arrays, in an ARVC family showing no mutations in any of the desmosomal genes. The CNVs analysis identified a heterozygous deletion of about $122 \mathrm{~kb}$ on chromosome $12 \mathrm{p} 11.21$, including the entire plakophilin-2 gene and shared by all affected family members. It was not listed on any of available public CNVs databases and was confirmed by quantitative real-time PCR. This is the first SNP array-based genome-wide study leading to the identification of a CNV segregating with the disease phenotype in an ARVC family. This result underscores the importance of performing additional analysis for possible genomic deletions/duplications in ARVC patients without point mutations in known disease genes.
\end{abstract} European Journal of Human Genetics (2013) 21, 1226-1231; doi:10.1038/ejhg.2013.39; published online 13 March 2013

Keywords: sudden death; cardiomyopathy; desmosome; plakophilin-2; gene deletion

\section{INTRODUCTION}

Arrhythmogenic right ventricular cardiomyopathy (ARVC) (MIM no. 107970) is an inherited cardiac disease characterized by fibro-fatty replacement of myocardial tissue, primarily affecting the right ventricle, even if the left ventricle is frequently involved. It is clinically characterized by the presence of high incidence of ventricular arrhythmias that can lead to sudden cardiac death, particularly in young people and athletes. ${ }^{1-3}$

To date, causative mutations have been identified mainly in genes encoding desmosomal proteins: plakoglobin $(J U P),{ }^{4,5}$ desmoplakin $(D S P),{ }^{6}$ plakophilin-2 $($ PKP2 $){ }^{7} \quad$ desmoglein-2 $\quad(D S G 2)^{8}$ and desmocollin-2 (DSC2). ${ }^{9}$ Additional mutations in extradesmosomal genes have been recognized as rare causes of ARVC or of clinical overlap syndromes mimicking ARVC. ${ }^{10-15}$ The emerging genetic profile of ARVC has fostered the view that desmosomal dysfunction may be the final common pathway underlying the disease.

The large majority of ARVC mutations identified so far are point mutations, either missense or splice-site variations or short indels. ${ }^{16-19}$

In a unique systematic study, applying Multiplex Ligationdependent Probe Amplification (MLPA) on 169 Dutch ARVC patients, large copy number variants involving PKP2 gene were detected in three $(1,8 \%)$ cases. ${ }^{19}$ Very recently, a deletion of the entire coding region of PKP2 gene (excluding exon 1) and a 7.9 Mb deletion of chromosome 12p12.1-p11.1 encompassing PKP2 exons 1-14 were identified in two ARVC cases, by chromosomal oligo-array analysis and/or MLPA. ${ }^{20}$
Conventional mutation screening in the desmosomal genes fails to detect causative mutations in about $50 \%$ of index cases, ${ }^{3}$ suggesting further genetic heterogeneity or presence of large deletions/ duplications involving known genes.

In the present study, we performed a genome-wide linkage study and a copy number variation (CNV) analysis aimed to identify novel disease gene and/or large pathogenic CNVs in an ARVC family negative for point mutations in desmosomal genes.

We report the first case of a PKP2 CNV detected by using highdensity SNPs array and segregating with the disease phenotype within the family.

\section{MATERIALS AND METHODS}

\section{Clinical study}

The proband was a male who was examined at the age of 46 . His threegeneration family consisted of 21 individuals ( 10 males and 11 females), aged from 23-83 years (Figure 1). After completing the proband diagnostic evaluation, all relatives have been approached for clinical examinations, independently of the presence of cardiac symptoms. All participating subjects gave written informed consent for clinical and genetic studies, according to the pertinent Italian legislation and in compliance with the Helsinki declaration.

The study protocol for clinical evaluation included a detailed personal/ family history, physical examination, 12-lead electrocardiography (ECG), signal averaged ECG (SAECG), 24-h Holter monitoring, two-dimensional and Doppler echocardiogram and stress test. According to the programmed clinical follow-up, the clinical evaluation (ECG, SAECG and echocardiogram) was

${ }^{1}$ Department of Biology, University of Padua, Padua, Italy; ${ }^{2}$ Department of Cardiac, Thoracic, and Vascular Sciences, University of Padua, Padua, Italy; ${ }^{3}$ Porto Conte Ricerche, Alghero, Italy; ${ }^{4}$ CNR-Institute of Neurosciences, University of Padova, Padova, Italy

*Correspondence: Dr A Rampazzo, Department of Biology, University of Padua, Via G. Colombo 3, Padua 35131, Italy. Tel: + 39 0498276208; Fax: + 39 0498276209; E-mail: alessandra.rampazzo@unipd.it

Received 5 November 2012; revised 1 February 2013; accepted 6 February 2013; published online 13 March 2013 


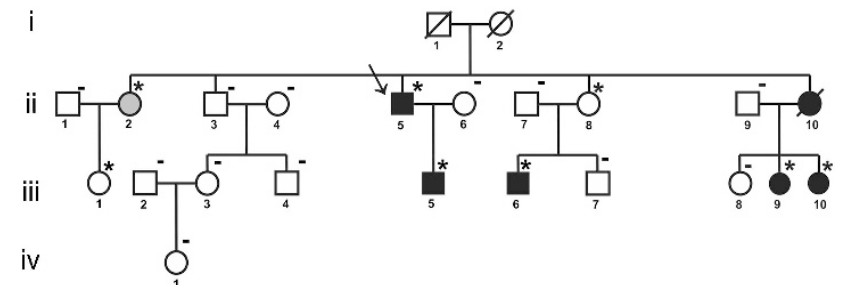

Figure 1 Pedigree of the ARVC family. Proband is indicated by the arrow. Circles denote females; squares denote males. Black and white symbols represent clinically affected and unaffected individuals, respectively; gray symbol indicates the subject with minor signs of the disease. Asterisks denote subjects carrying the heterozygous PKP2 deletion; traits indicate a normal gene copy number.

performed every 6 months in subjects showing clinical signs of the disease and every 12 months in the remaining family members. The clinical follow-up was performed by the same clinical staff, composed of two physicians. The duration of the follow-up of the proband was 23 years; the mean follow-up duration of family members was $19 \pm 5$ years (range 13-22 years).

The original diagnosis of ARVC in the proband was put forward in 1995 , according to 1994 International Task Force criteria. ${ }^{21}$ Then, after the publication of the 2010 modified Task Force criteria, ${ }^{22}$ all subjects were reevaluated and both proband and affected family members fulfill the current diagnostic criteria. ${ }^{22}$

Subjects were considered affected by a mild, moderate or severe form of the disease on the basis of echocardiographic evaluation. ${ }^{23}$ In particular, patients were subdivided by echocardiography investigations into three groups according to right ventricular (RV) end-diastolic volume (RVEDV): (1) mild form-a normal or slightly increased RVEDV $\left(<75 \mathrm{ml} / \mathrm{m}^{2}\right)$ with localized hypokinetic or akinetic areas, in the presence or absence of trabecular disarrangements and thickened, hyperechogenic or dense moderator band; (2) moderate form-a $75-120 \mathrm{ml} / \mathrm{m}^{2}$ RVEDV with localized hypokinetic or akinetic and/or dyskinetic areas and/or trabecular disarrangement and thickened, hyperechogenic or dense moderator band; (3) severe form-an RVEDV $\geq 120 \mathrm{ml} / \mathrm{m}^{2}$ with widespread akinetic and/or dyskinetic areas of the $\mathrm{RV}$ and diastolic bulging.

\section{Mutation screening}

The coding regions of PKP2, DSP, DSG2, DSC2 and JUP genes were screened for mutations in the index case. Each sequence was amplified from genomic DNA by PCR assay. Primer sequences are available on request. The analysis was performed by Denaturing High Performance Liquid Chromatography (DHPLC, Transgenomic, NE, USA) and direct sequencing.

\section{SNP array genotyping}

Individual genotyping was performed using the high-density SNP marker set Infinium HumanCNV370-Duo BeadChip (Illumina, San Diego, CA, USA), which includes 370404 markers. For each family member, $200 \mathrm{ng}$ of DNA was analyzed on Illumina Iscan System (Illumina, San Diego, CA, USA), according to the manufacturer's protocol.

\section{Linkage analysis}

The raw genotyping data were processed by Plink software v1.07. ${ }^{24}$ Genetic distances between markers were determined using the deCODE genetic map ${ }^{25}$ and base pair positions, as well as the marker's order, were obtained from the human reference sequence assembly (hg19 release, February 2009) (http:// genome.ucsc.edu)

All allele calls were checked for Mendelian inconsistencies by Plink. The reliability of the called genotypes was evaluated based on the call rate, removing SNPs with call rates $<95 \%$. To avoid potential bias caused by rare alleles, only SNPs with minimum allele frequency $>1 \%$ were considered for the analysis. SNPs in the same physical position or mapped on multiple loci were not included in the analysis.
The genome-wide linkage study was performed with GeneHunter software version $2.1,{ }^{26}$ through the graphical user interface EasyLinkage Plus v5.08, ${ }^{27}$ using a parametric multipoint approach.

An autosomal dominant model with 0.0001 disease allele frequency and 0.02 phenocopy rate was specified. Taking into account the incomplete penetrance of the disease, a conservative 'affected-only' approach was adopted. All affected individuals were included in the analysis and all other subjects not fulfilling the current ARVC diagnostic criteria were coded as unknown phenotype. This approach provides a safeguard against false negative findings due to incorrect specification of the penetrance model.

For all regions showing positive LOD score values, the haplotypes of markers were reconstructed by GeneHunter using Viterbi algorithm and displayed by HaploPainter program. ${ }^{28}$

\section{CNVs analysis}

PennCNV algorithm ${ }^{29}$ was used to infer the most likely copy number state from the signal intensity data at each SNP, represented as normalized total fluorescent intensity values from both alleles ( $\log \mathrm{R}$ Ratio) and normalized relative ratio of fluorescent signals between two alleles (B Allele Frequency).

The gc-model file was included in the analysis to correct for 'genomic waves' in signal intensities due to highly GC-rich regions and affecting the accuracy of CNVs calling. ${ }^{30}$

Quality control analysis was performed by PennCNV software (http:// www.neurogenome.org/cnv/penncnv), considering the following criteria: SD for autosomal $\log$ R Ratio $>0.28$, median B Allele Frequency $>0.55$ or $<0.45$, $B$ Allele Frequency drift $>0.002$ and waviness factor $>0.05$. In order to reduce the number of false positives, CNV calls were required to include at least five probes and to span genomic regions $>10 \mathrm{~Kb}$ in size.

\section{Quantitative real-time PCR (qPCR)}

In order to confirm the presence of the PKP2 gene deletion in eight family members, quantitative real-time PCR was performed using primers specific for exons 2, 7 and 13 of the PKP2 gene (Table 1).

To normalize the target gene copy number, beta-actin (ACTB) and TATAbinding protein $(T B P)$ genes were used as internal references (Table 1).

Genomic DNA from eight subjects carrying the PKP2 gene deletion, three family members with a normal gene copy number (based on PennCNV calls) and a control sample was amplified by real-time PCR on an ABI PRISM 7900HT Fast Sequence Detection System (Applied Biosystems, Foster City, CA, USA).

qPCR reactions were carried out in triplicate for each sample, in a final volume of $10 \mu \mathrm{l}$, containing $10 \mathrm{ng}$ of genomic DNA, 2X SYBR Green PCR Master Mix (DyNAmoTM HS SYBR Green qPCR Kit, Finnzymes, Espoo, Finland), and the appropriate concentrations of each primer. Amplification conditions consisted of initial denaturation step at $95^{\circ} \mathrm{C}$ for $10 \mathrm{~min}$, followed by 40 cycles at $95^{\circ} \mathrm{C}$ for $15 \mathrm{~s}$ and at $62{ }^{\circ} \mathrm{C}$ for $1 \mathrm{~min}$. The $\Delta \Delta \mathrm{Ct}$ quantification method was used to determine the relative gene copy number in each sample. Validation experiments were performed using the standard curve method with five serial dilutions of genomic DNA from control subjects. PCR efficiencies (E) for all exons were calculated according to: $\mathrm{E}=\left(10^{(-1 / \text { slope })}-1\right) \times 100$, where $\mathrm{E}=100$ corresponds to $100 \%$ efficiency. Amplification efficiencies were identical and the relative gene copy number was calculated by $2^{-\Delta \Delta C t}$, as previously described. ${ }^{31}$

\section{RESULTS}

\section{Linkage analysis}

Genetic analysis of coding regions of PKP2, DSP, DSG2, DSC2 and JUP genes excluded the presence of point mutations in the proband's DNA. Therefore, a genome-wide scan was performed to exclude linkage to known ARVC loci and to eventually search for novel ARVC genes.

Linkage to DSP, DSG2, DSC2, DES, LMNA genes and ARVD3 locus was excluded ( $\mathrm{pLOD}<-2.0$ ) (data not shown).

Multipoint linkage analysis, using an 'affected-only' approach, gave a maximum parametric (p)LOD score of 1.17 , formally not significant 
Table 1 Sequences of primers used for real-time quantitative PCR amplification

\begin{tabular}{lrrll}
\hline \multicolumn{5}{c}{ Amplicon } \\
Gene & Exon & size $(b p)$ & Primer forward 5'-3' & Primer reverse 5'-3' \\
\hline PKP2 & 2 & 127 & TTTAATATTTGCTTGATTGCAG & TGTCATAGGTTTTAGGAACAGG \\
& 7 & 140 & TTTTCAGCGGTCATTTTGG & TCTCCGTCAGCGTAAGCAAT \\
& 13 & 113 & AGCAGTTGAGGAGCGAGAG & TTGTTGGAGGCATAGCTGAA \\
ACTB & 4 & 59 & CGAGCGCGGCTACAGCTT & CCTTAATGTCACGCACGATT \\
TBP & 2 & 106 & CTGTTTCTTGGCGTGTGAG & CGCTGGACTCGTCTCACTA \\
\hline
\end{tabular}
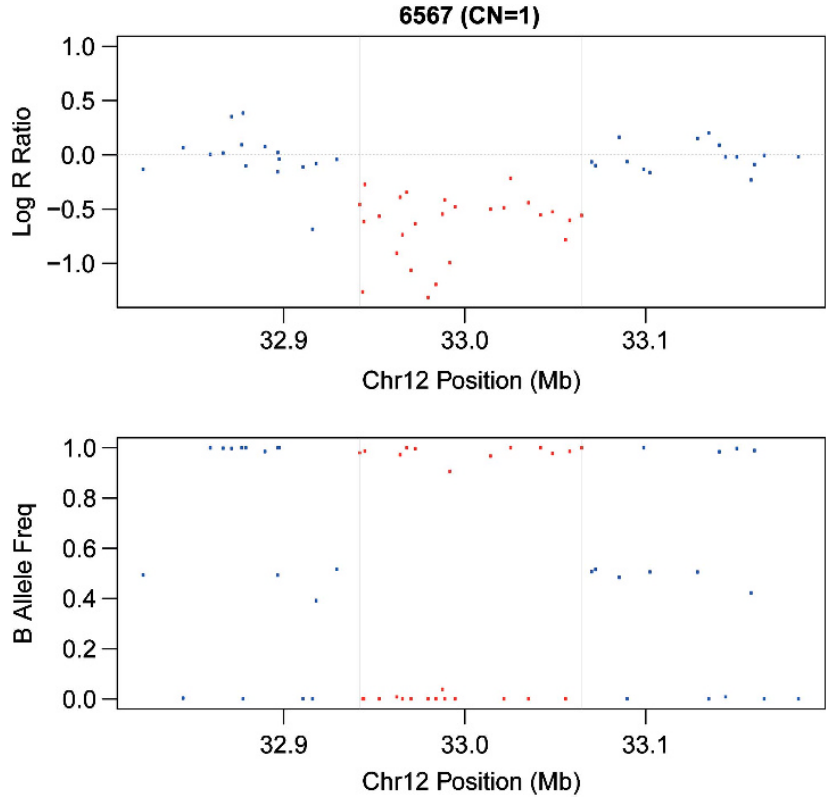

Figure 2 Results of CNVs analysis on chromosome 12p11.21 showing the PKP2 gene deletion. Log R Ratios (top) and B allele frequencies (bottom) are plotted against chromosome 12 position (32822606-33 $184590 \mathrm{bp}$ ). Red dots denote SNPs included in the deleted region, blue dots indicate markers outside the deletion. The length of the deleted region is displayed as distance between the two gray vertical lines and included 26 SNPs. Hemizygosity appears as a drop in log R Ratio (red dots below 0.0) and as a missing in heterozygote SNP calls (lack of dots at 0.5 ).

but close to the maximum simulated LOD score of 1.21, for 11 chromosomal regions (2q14.2-q31.1; 4p16.3-p15.33; 4q23-q25; 8p22-p21.2; 8q22.1-q22.3; 9q33.3-q34.2; 10p15.3-p15.1; 12p12.3q13.13; 13q33.3-q34; 16p13.13-p12.1; 21q22.13-q22.3).

Among them, the region on chromosome 12p12.3-q13.3 included the PKP2 gene. As no point mutations in PKP2 gene were identified in the index case, a CNVs analysis was performed to evaluate the presence of large genomic rearrangements (deletions or duplications $>1 \mathrm{~kb}$ in size), shared by all affected family members.

\section{CNVs analysis}

The whole SNP genotype data set of each subject was analyzed by PennCNV software and a total of $511 \mathrm{CNV}$ s were identified.

Among all the detected CNVs, a heterozygous deletion of $122.548 \mathrm{~Kb}$ on chromosome 12p11.21 (Figure 2) was identified in all affected family members (II-5; III-5; III-6; III-9; III-10), two healthy subjects (II-8; III-1) and one individual (II-2) with minor signs of disease, not fulfilling the diagnostic 2010 Task Force criteria (Figure 1). The deletion spans the region between SNPs rs6488089 (chromosome position: $32942278 \mathrm{bp}$ ) and rs7295486 (chromosome position: $33064825 \mathrm{bp}$ ) and includes 26 markers. It encompassed the entire PKP2 gene and was not present in any of the available databases: 1000 Genomes Project (http://www.1000genomes.org), Database of Genome Variants (http://projects.tcag.ca/variation) and Decipher (http://decipher.sanger.ac.uk).

No other CNVs shared by all affected family members were detected.

Gene dosage performed by $\mathrm{qPCR}$ with primers specific for PKP2 exons 2, 7 and 13 confirmed the deletion in all eight subjects, as predicted by PennCNV software. Real-time PCR results indicated a relative copy number of about 0.5 for each PKP2 exon in the heterozygous deletion carriers, compared with three family members with a normal gene copy number (based on PennCNV calls) and one external control sample (Figure 3).

\section{Clinical findings}

The clinical features of the proband and family members carrying the PKP2 gene deletion are summarized in Table 2.

Male proband II-5 (Figure 1) was examined at the age of 46 years, due to effort dyspnea. Two-dimensional echocardiogram revealed the presence of RV dilation and hypokinesia, suggesting a mild form of ARVC. At the age of 52 years several episodes of polymorphic non sustained ventricular tachycardia were recorded at 24-h Holter ECG, thus a therapy with propaphenone was prescribed. At that time, basal ECG showed the presence of negative T waves in V1-V3 with epsilon wave in right precordial leads in the absence of complete right bundle-branch block (RBBB) and epsilon wave in right precordial leads. Late potentials were detected at 25, 40 and 80 filter setting. Echocardiogram showed normal left ventricle (LV) parameters, severe RV dilation (end-diastolic area-EDA: $42 \mathrm{~cm}^{2}$ ), reduction of the RV fractional area change (FAC: $30 \%$ ), apical and subtricuspid akinesia and presence of apical sacculations. The clinical diagnosis of ARVC was put forward on the basis of the current Task Force criteria.

After 4 years, echocardiogram revealed a LV involvement with a reduced ejection fraction (EF: 47\%) and inferior wall hypokinesia. The 24-h Holter ECG demonstrated the presence of isolated premature ventricular beats with RBBB and left bundle-branch block (LBBB) morphologies and superior axis deviation. In the last year, the patient complained chest pain and coronarography showed normal coronary arteries. $\mathrm{He}$ is currently undergoing an antiarrhythmic therapy with propafenone and beta-blockers.

Subject III-6 has a severe form of the disease without LV involvement. He showed a sustained VT with LBBB morphology at the age of 21; 12-lead ECG demonstrated the presence of negative $\mathrm{T}$ waves in V1-V2 and biphasic in V3 (Figure 4). Endomyocardial biopsy was performed and cardiomyopathic changes were found at histological examination, associated with inflammatory infiltrates, although never reaching the diagnostic criteria in terms of fibro-fatty replacement due to the septal site of sampling.

Among the other deletion carriers, subject III-9 shows a severe form of the disease, subjects III- 5 and III- 10 a moderate and a mild form, respectively, II-2 shows premature ventricular beats with LBBB morphology at 24-h Holter ECG $(<500 / 24 \mathrm{~h})$, thus not fulfilling the current diagnostic criteria, whereas II- 8 and III-1 show negative clinical signs. 


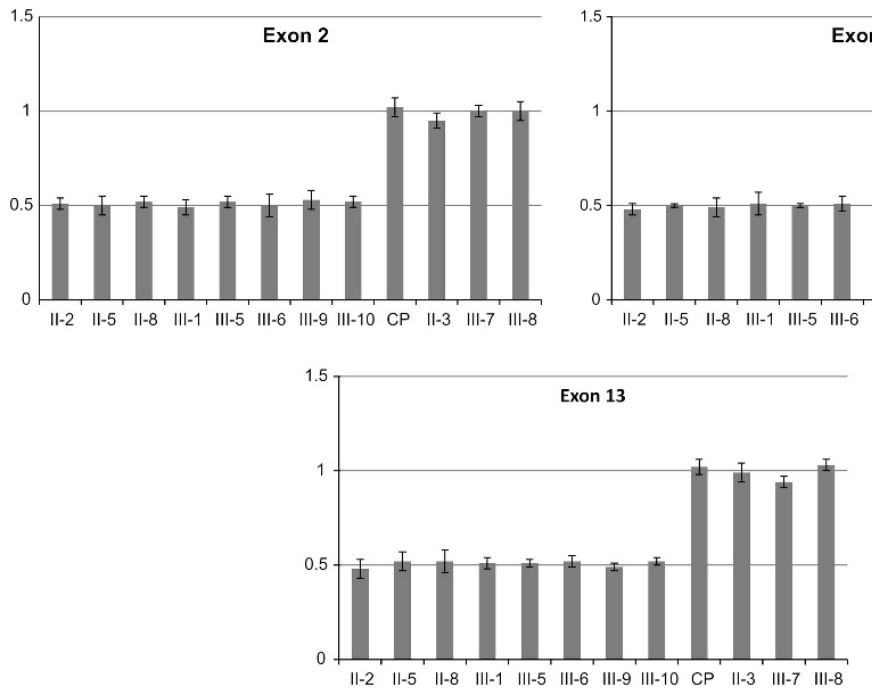

Figure 3 PKP2 gene dosage by quantitative real-time PCR in a control individual (CP) and family members. The target gene copy number was normalized to the reference gene TATA-binding protein (TBP). Each experiment was done in triplicate. Error bars represent SD of replicates. Similar results (not showed) were obtained using $\beta$-actin (ACTB) as internal reference gene.

Table 2 Clinical data of proband and family members carrying the PKP2 gene deletion

\begin{tabular}{|c|c|c|c|c|c|c|c|c|c|c|c|c|c|}
\hline \multirow[b]{2}{*}{ Subject } & \multirow[b]{2}{*}{ Sex } & \multirow[b]{2}{*}{$\begin{array}{c}\text { Age at diagnosis/ } \\
\text { investigation }\end{array}$} & \multicolumn{2}{|c|}{ Family history } & \multicolumn{2}{|l|}{ 12-lead ECG } & \multicolumn{3}{|c|}{ Arrhythmias } & \multicolumn{2}{|c|}{ RV size/function } & \multirow[b]{2}{*}{$\begin{array}{c}\text { LV } \\
\text { involvement }\end{array}$} & \multirow[b]{2}{*}{$\begin{array}{c}\text { Diagnostic } \\
\text { criteria } 2010\end{array}$} \\
\hline & & & Major & Minor & $\begin{array}{l}\text { Negative } T \text { waves in } \\
\text { right precordial leads }\end{array}$ & $\begin{array}{c}\text { Epsilon } \\
\text { wave }\end{array}$ & SAECG & $\begin{array}{l}P V C s>500 / \\
24-h N S V T\end{array}$ & $\begin{array}{l}\angle B B B \\
S V T\end{array}$ & Major & Minor & & \\
\hline 11,5 & M & 52 & - & - & V1-V3 & + & + & + & - & + & - & + & $3 \mathrm{M}, 1 \mathrm{~m}$ \\
\hline III,5 & $M$ & 24 & + & - & - & - & + & + & - & - & + & - & $1 \mathrm{M}, 3 \mathrm{~m}$ \\
\hline III,6 & M & 21 & + & - & V1-V2 & - & + & + & + & + & - & - & $2 \mathrm{M}, 2 \mathrm{~m}$ \\
\hline III,9 & $\mathrm{F}$ & 28 & + & - & V1-V3 & - & - & + & - & + & - & - & $2 \mathrm{M}, 2 \mathrm{~m}$ \\
\hline III,10 & $\mathrm{F}$ & 32 & + & - & - & - & - & + & - & - & + & - & $1 \mathrm{M}, 2 \mathrm{~m}$ \\
\hline $\mathrm{II}, 2$ & $\mathrm{~F}$ & 68 & + & - & - & - & - & - & - & - & - & - & $1 \mathrm{M}$ \\
\hline 11,8 & $\mathrm{~F}$ & 60 & + & - & - & - & - & - & - & - & - & - & $1 \mathrm{M}$ \\
\hline III,1 & $\mathrm{F}$ & 31 & + & - & - & - & - & - & - & - & - & - & $1 \mathrm{M}$ \\
\hline
\end{tabular}

Abbreviations: M, major criteria; m, minor criteria; NSVT, non sustained ventricular tachycardia; LV, left ventricular; RV, right ventricular; SAECG, signal-averaged ECG; SVT, sustained ventricular tachycardia.

\section{DISCUSSION}

Variants in PKP2 gene are relatively common among ARVC patients, as they have been identified in $7-51 \%$ of cases with autosomal dominant inheritance pattern. $3,7,16-19$

Frameshift and nonsense mutations account for 35\% (79 out of 224) of PKP2 genetic alterations identified in ARVC patients (ARVD/ C Genetic Variants database; http://www.arvcdatabase.info/).

In the present study, we report the first case of $\mathrm{CNV}$ identified in an ARVC family using high-density SNPs arrays.

A heterozygous deletion of about $122.5 \mathrm{~Kb}$ on chromosome 12 p11.21, encompassing the entire PKP2 gene, was detected in all affected family members. It was not present in any of available public CNVs databases and was confirmed by quantitative real-time PCR. Overall, these data support the pathogenic nature of the deletion, which is expected to determine haploinsufficiency. This effect may be similar to that of a premature termination codon mutation with subsequent nonsense-mediated decay degradation of the PKP2 mRNA.
The occurrence of the deletion also in one family member with minor signs of the disease, not fulfilling the 2010 diagnostic Task Force criteria, and in two healthy relatives is consistent with the incomplete penetrance of ARVC mutations. Genetic, epigenetic and environmental factors could influence disease expression and mutation penetrance. Similarly to that observed in hypertrophic cardiomyopathy, ${ }^{32}$ dilated cardiomyopathy ${ }^{33}$ and long-QT syndrome, ${ }^{34}$ we can speculate that common sequence variants, modifying the effects of the primary mutation, may influence penetrance and phenotypic manifestations in this family. Moreover, allelic asymmetries, as allele-specific gene expression, allele-specific DNA methylation and allele-specific transcription factor binding, could influence the expression ratio of the two alleles. ${ }^{35}$ All these types of allelic asymmetries are tissuespecific and the extent of the allelic bias can vary substantially among individuals; accordingly, we can speculate that an allelic imbalance at the PKP2 locus may influence the disease susceptibility in this family.

Interestingly, the telomeric end of the deletion identified in our study lies about $1.4 \mathrm{~kb}$ upstream from a $2 \mathrm{~kb}$ low-copy repeat (LCR) 
a

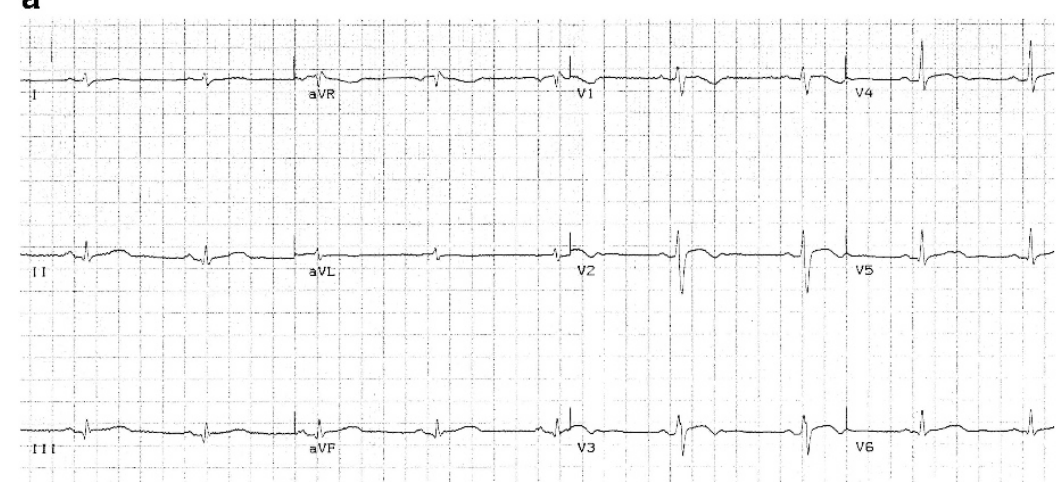

b

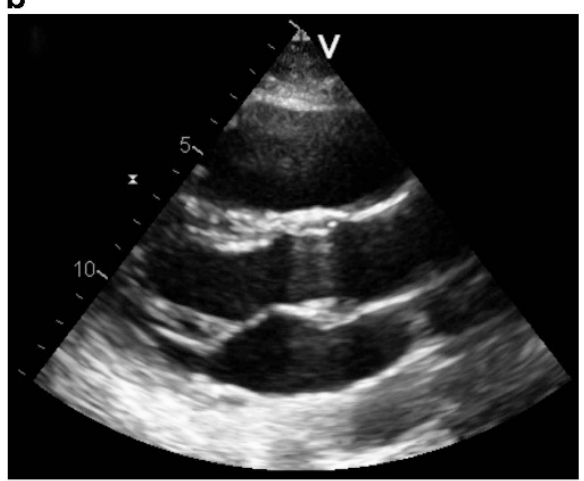

C

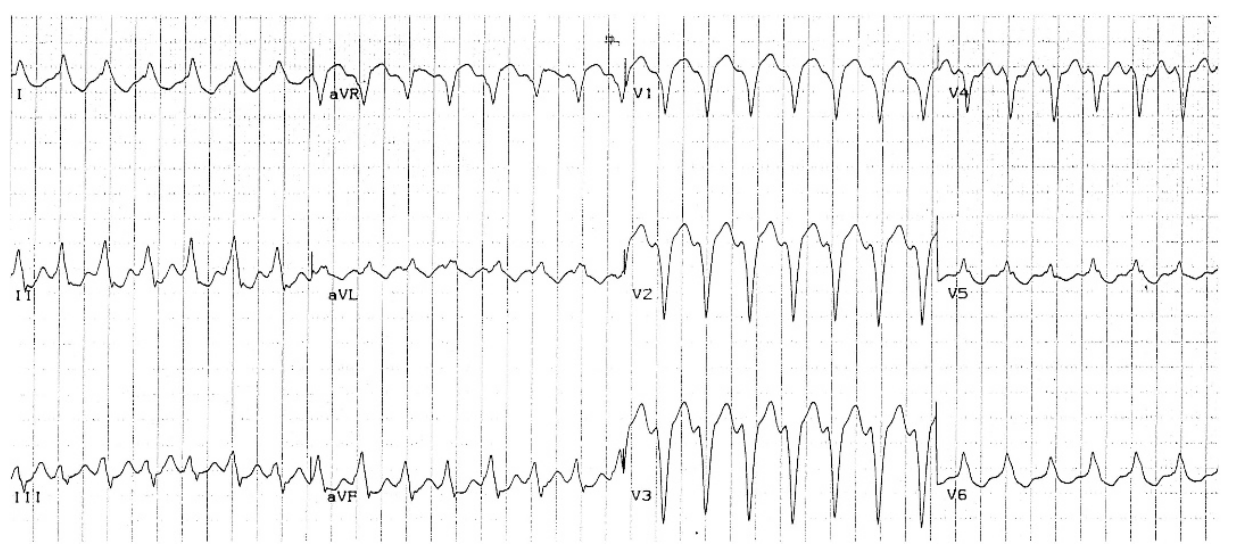

Figure 4 Instrumental findings of patient III-6 at the time of diagnosis. (a) 12-lead ECG showing sinus rhythm, negative T waves in V1-V2 and biphasic in V3; (b) 2D-echocardiogram (parasternal long axis view) with evidence of a dilated right ventricle (RVOT: 19,2 mm/m²); (c) sustained VT tachycardia with LBBB morphology and right axis deviation.

(genomic position chr12:32943683-32945665) and a second LCR of similar size is located more than $26 \mathrm{Mb}$ upstream (genomic position chr12:6589220-6591352). LCRs or segmental duplications are DNA fragments that range from $1-400 \mathrm{~kb}$ in length, occur at more than one site within the genome, and typically share a high level (>90\%) of sequence identity. ${ }^{36}$ LCRs can cause genomic instability and either mediate or stimulate both recurrent and non recurrent $\mathrm{CNV}$ formation. The predominant molecular mechanism underlying recurrent genomic rearrangements is non allelic homologous recombination (NAHR) between large directly oriented LCRs with a DNA sequence identity greater than $97 \%$ and located at a distance less than $\sim 10 \mathrm{Mb}$ from each other. NAHR leads to misalignment of chromosomes or chromatids during meiosis resulting in deletions or reciprocal duplications of the genomic segment between them. ${ }^{36}$ However, PKP2 gene is located downstream from both LCRs on chromosome 12; accordingly, LCRs-based NAHR cannot account for formation of PKP2 CNVs.

It can be hypothesized that PKP2 deletions may arise by non recurrent rearrangements due to replication-based mechanisms of DNA repair, as fork stalling and template switching and microhomology-mediated break-induced replication, that utilize nucleotide microhomology at the rearrangement breakpoints and were recently proposed to be responsible for formation of non recurrent CNVs and complex genomic rearrangements. ${ }^{37}$

To date, large genomic deletions involving PKP2 gene were detected with a relatively low frequency in ARVC patients. ${ }^{19,20}$ The identification of a $\mathrm{CNV}$ segregating with the disease in this family emphasizes the importance of complementing the conventional mutation screening in ARVC genes with other approaches able to detect possible structural variations.

Moreover, a genome-wide CNVs analysis, using high-resolution SNP arrays, may be useful for identifying CNVs in cardiomyopathyrelated genes, introns, regulatory elements, and coding regions of the known ARVC genes, not covered by the common MLPA kits, as well as in novel disease genes lying in relatively unstable genomic regions.

Recent studies described the involvement of rare $\mathrm{CNV}$ s in the occurrence of long QT syndrome ${ }^{38,39}$ and dilated cardiomyopathy, ${ }^{40}$ suggesting that genomic structural variations may underlie inherited cardiomyopathies, although the exact contribution of CNVs to these disorders remains unknown.

CNVs could constitute $20 \%$ of mutations involved in Mendelian diseases; ${ }^{41}$ this is of particular relevance to ARVC, where only $\sim 50 \%$ of genetic causes have been identified. Further large-scale studies on patients and family members are required to evaluate the frequency of CNVs in ARVC genes and to establish a genotype-phenotype correlation.

\section{CONFLICT OF INTEREST}

The authors declare no conflict of interest.

\section{ACKNOWLEDGEMENTS}

We thank Mrs Paola Marcon for her help in collecting ARVC families. This work was supported by Italian Telethon Foundation, Rome GGP09293, Fondazione CARIPARO, Padua and Association Française contre les Myopathies (AFM), Paris, grant 14776 to AR. 
1 Thiene G, Nava A, Corrado D, Rossi L, Pennelli N: Right ventricular cardiomyopathy and sudden death in young people. N Engl J Med 1988; 318: 129-133.

2 Nava A, Thiene G, Canciani B et al: Familial occurrence of right ventricular dysplasia: a study involving nine families. J Am Coll Cardiol 1988; 12: 1222-1228.

3 Basso C, Bauce B, Corrado D, Thiene G: Pathophysiology of arrhythmogenic cardiomyopathy. Nat Rev Cardiol 2011; 9: 223-233.

4 McKoy G, Protonotarios N, Crosby A et al: Identification of a deletion in plakoglobin in arrhythmogenic right ventricular cardiomyopathy with palmoplantar keratoderma and woolly hair (Naxos disease). Lancet 2000; 355: 2119-2124.

5 Asimaki A, Syrris P, Wichter T, Matthias P, Saffitz JE, McKenna WJ: A novel dominant mutation in plakoglobin causes arrhythmogenic right ventricular cardiomyopathy. Am J Hum Genet 2007; 81: 964-973.

6 Rampazzo A, Nava A, Malacrida S et al: Mutation in human desmoplakin domain binding to plakoglobin causes a dominant form of arrhythmogenic right ventricular cardiomyopathy. Am J Hum Genet 2002; 71: 1200-1206.

7 Gerull B, Heuser A, Wichter Tet al: Mutations in the desmosomal protein plakophilin-2 are common in arrhythmogenic right ventricular cardiomyopathy. Nat Genet 2004; 36: $1162-1164$.

8 Pilichou K, Nava A, Basso C et al: Mutations in desmoglein-2 gene are associated with arrhythmogenic right ventricular cardiomyopathy. Circulation 2006; 113: 1171-1179.

9 Syrris $\mathrm{P}$, Ward D, Evans $\mathrm{A}$ et al: Arrhythmogenic right ventricular dysplasia/cardiomyopathy associated with mutations in the desmosomal gene desmocollin-2. Am J Hum Genet 2006; 79: 978-984

10 Tiso N, Stephan DA, Nava A et al: Identification of mutations in the cardiac ryanodine receptor gene in families affected with arrhythmogenic right ventricular cardiomyopathy type 2 (ARVD2). Hum Mol Genet 2001; 10: 189-194.

11 Beffagna G, Occhi G, Nava A et al: Regulatory mutations in transforming growth factorbeta3 gene cause arrhythmogenic right ventricular cardiomyopathy type 1. Cardiovasc Res 2005; 65: 366-373.

12 Merner ND, Hodgkinson KA, Haywood AF et al: Arrhythmogenic right ventricular cardiomyopathy type 5 is a fully penetrant, lethal arrhythmic disorder caused by a missense mutation in the TMEM43 gene. Am J Hum Genet 2008; 82: 809-821.

13 Klauke B, Kossmann S, Gaertner A et al: De novo desmin-mutation N116S is associated with arrhythmogenic right ventricular cardiomyopathy. Hum Mol Genet 2010; 19: 4595-4607.

14 Taylor M, Graw S, Sinagra G et al: Genetic variation in titin in arrhythmogenic right ventricular cardiomyopathy-overlap syndromes. Circulation 2011; 124: 876-885.

15 Quarta G, Syrris P, Ashworth M et al: Mutations in the Lamin A/C gene mimic arrhythmogenic right ventricular cardiomyopathy. Eur Heart J 2012; 33: 1128-1136.

16 Fressart V, Duthoit G, Donal E et al: Desmosomal gene analysis in arrhythmogenic right ventricular dysplasia/cardiomyopathy: spectrum of mutations and clinical impact in practice. Europace 2010; 12: 861-868.

17 Bauce B, Nava A, Beffagna $G$ et al: Multiple mutations in desmosomal proteins encoding genes in arrhythmogenic right ventricular cardiomyopathy/dysplasia. Heart Rhythm 2010; 7: 22-29.

18 Quarta G, Muir A, Pantazis A et al: Familial evaluation in arrhythmogenic right ventricular cardiomyopathy: impact of genetics and revised task force criteria. Circulation 2011: 123: 2701-2709.

19 Cox MG, van der Zwaag PA, van der Werf $\mathrm{C}$ et al: Arrhythmogenic right ventricular dysplasia/cardiomyopathy: pathogenic desmosome mutations in index-patients predict outcome of family screening: Dutch arrhythmogenic right ventricular dysplasia/cardiomyopathy genotype phenotype follow-up study. Circulation 2011: 123: 2690-2700.

20 Roberts JD, Herkert JC, Rutberg J et al: Detection of genomic deletions of PKP2 in Arrhythmogenic Right Ventricular Cardiomyopathy. Clin Genet 2012; e-pub ahead of print 13 August 2012.
21 McKenna WJ, Thiene G, Nava A et al: Diagnosis of arrhythmogenic right ventricular dysplasia/cardiomyopathy: Task Force of the Working Group Myocardial and Pericardial Disease of the European Society of Cardiology and of the Scientific Council on Cardiomyopathies of the International Society and Federation of Cardiology. Br Heart $\mathrm{J}$ 1994; 71: 215-218.

22 Marcus FI, McKenna WJ, Sherrill D et al: Diagnosis of arrhythmogenic right ventricular cardiomyopathy/dysplasia: proposed modification of the Task Force Criteria. Eur Heart J 2010; 31: 806-814.

23 Nava A, Bauce B, Basso $C$ et al: Clinical profile and long term follow-up of 37 families with arrhythmogenic right ventricular cardiomyopathy. J Am Coll Cardiol 2000; 36: 2226-2233.

24 Purcell S, Neale B, Todd-Brown K et al: PLINK: a toolset for whole-genome association and population-based linkage analysis. Am J Hum Genet 2007; 81: 559-575.

25 Kong A, Gudbjartsson DF, Sainz J et al: A high-resolution recombination map of the human genome. Nat Genet 2002; 31: 241-247.

26 Kruglyak L, Daly MJ, Reeve-Daly MP, Lander ES: Parametric and nonparametric linkage analysis: a unified multipoint approache. Am J Hum Genet 1996; 58: 1347-1363.

27 Lindner TH, Hoffmann K: EasyLINKAGE: a PERL script for easy and automated two-/ multi-point linkage analysis. Bioinformatics 2005; 21: 405-407.

28 Thiele H, Nurnberg P: HaploPainter: a tool for drawing pedigrees with complex haplotypes. Bioinformatics 2005; 21: 1730-1732.

29 Wang K, Li M, Hadley D et al: PennCNV: an Integrated hidden Markov model designed for high-resolution copy number variation detection in whole-genome SNP genotyping data. Genome Res 2007; 17: 1665-1674.

30 Diskin SJ, Li M, Hou C et al: Adjustment of genomic waves in signal intensities from whole-genome SNP genotyping platforms. Nucleic Acids Res 2008; 36: e126.

31 Livak KJ, Schmittgen TD: analysis of relative gene expression data using real-time quantitative PCR and the 2(-Delta Delta C(T)) method. Methods 2001; 25: 402-408.

32 Rai TS, Dhandapany PS, Ahluwalia TS et al: ACE I/D polymorphism in Indian patients with hypertrophic cardiomyopathy and dilated cardiomyopathy. $\mathrm{Mol}$ Cell Biochem 2008; 311: 67-72.

33 Arvanitis DA, Sanoudou D, Kolokathis F et al: The Ser96Ala variant in histidine-rich calcium-binding protein is associated with life-threatening ventricular arrhythmias in idiopathic dilated cardio- myopathy. Eur Heart J 2008; 29: 2514-2525.

34 Crotti L, Lundquist AL, Insolia R et al: KCNH2-K897T is a genetic modifier of latent congenital long-QT syndrome. Circulation 2005; 112: 1251-1258.

35 Tycko B, Allele-specific DNA: Methylation: beyond imprinting. Hum Mol Genet 2010; 19: R210-R220.

36 Stankiewicz P, Lupski JR: Structural variation in the human genome and its role in disease. Annu Rev Med 2010; 61: 437-455.

37 Hastings PJ, Lupski JR, Rosenberg SM, Ira G: Mechanisms of change in gene copy number. Nat Rev Genet 2009; 10: 551-564.

38 Eddy CA, MacCormick JM, Chung SK et al: Identification of large gene deletions and duplications in KCNQ1 and $\mathrm{KCNH} 2$ in patients with long QT syndrome. Heart Rhythm 2008; 5: 1275-1281.

39 Barc J, Briec F, Schmitt S et al: screening for copy number variation in genes associated with the long QT syndrome. J Am Coll Cardiol 2011; 57: 40-47.

40 Norton N, Li D, Rieder MJ et al: Genome-wide studies of copy number variation and exome sequencing identify rare variants in BAG3 as a cause of dilated cardiomyopathy. Am J Hum Genet 2011; 88: 273-282.

41 Botstein D, Risch N: Discovering genotypes underlying human phenotypes: past successes for mendelian disease, future approaches for complex disease. Nat Genet 2003; 33: 228-237. 